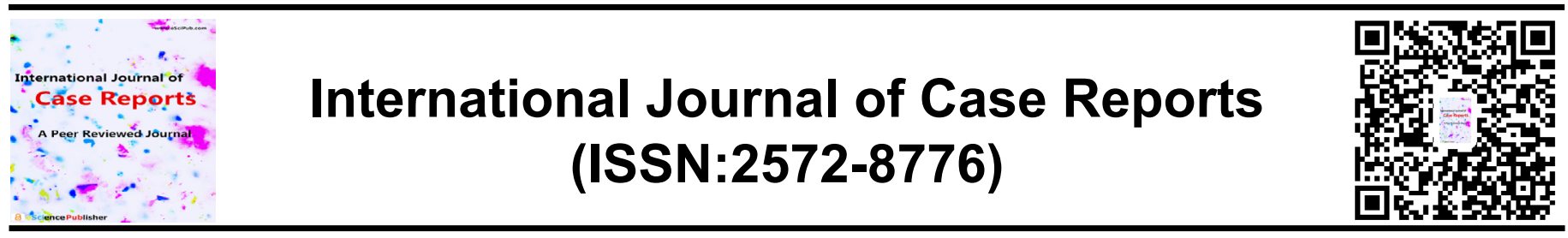

\title{
Prepyloric Diverticulum in a Nigerian: Case Report of a Rare Finding
}

\section{Aderemi Oluyemi $^{1+}$, Dayo Ajayi ${ }^{2}$}

${ }^{1}$ ReMay Consultancy \& Medical Services, Ikeja, Lagos, Nigeria.

${ }^{2}$ Renaissance Medical Center, Victoria Island, Lagos, Nigeria.

\section{ABSTRACT}

Diverticulae are uncommonly encountered in the stomach. They can be congenital or acquired- the latter variety being the less frequent of the two. This article details one such rare case from Lagos, Nigeria and discusses certain aspects of its clinical and endoscopic presentation. We present a 59-year old with a background history of non-steroidal anti-inflammatory drugs abuse presented with hematemesis and melena and was in hemorrhagic shock. After stabilization, the gastroscopy findings were of a gastric diverticulum (GD) in the anterior prepyloric wall. There was a Forrest Ilb ulcer in the GD's wall with surrounding erythema and edema. The patient was successfully managed medically and conservatively and with good long term results.

Keywords: gastric diverticulum; Nigeria; gastroscopy; ulcer.
*Correspondence to Author: Aderemi Oluyemi

ReMay Consultancy \& Medical Services, Ikeja, Lagos, Nigeria.

\section{How to cite this article:}

Aderemi Oluyemi, Dayo Ajayi. Prepyloric Diverticulum in a Nigerian: Case Report of a Rare Finding International Journal of Case Reports, 2020 4:134

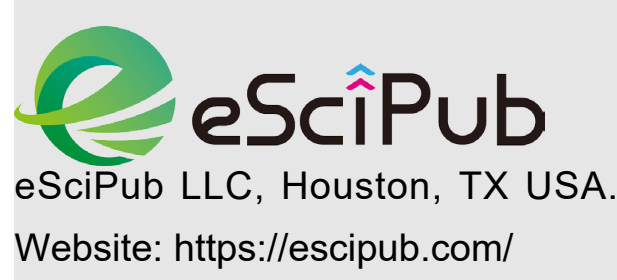




\section{Introduction}

A gastric diverticulum (GD) is an outpouching of the lining of the stomach. They are classified after Akerlund as congenital and acquired ${ }^{1}$. The congenital GD is a 'true diverticulum' and involves all layers of the stomach. They constitute about three quarters of all GDs are located close to the oesophagogastric junction.

Acquired gastric diverticulae in contrast are 'pseudodiverticulae' (as they lack the mucosa or serosal layers), are less common and typically located in the antrum. While the former types are usually incidental findings with little or no symptoms, these acquired ones usually present with a background history of other gastrointestinal (GIT) pathology, such as peptic ulcer disease, malignancy, pancreatitis, or gastric outlet obstruction ${ }^{2}$.

The stomach is the least common site for diverticulae in the gastrointestinal tract. The estimated prevalence figures of this rare phenomenon range from $0.01 \%$ to $0.26 \% 3,4,5$. The site of predilection is the fundus and only few reports have been documented in the more distal lower regions ${ }^{6}$.

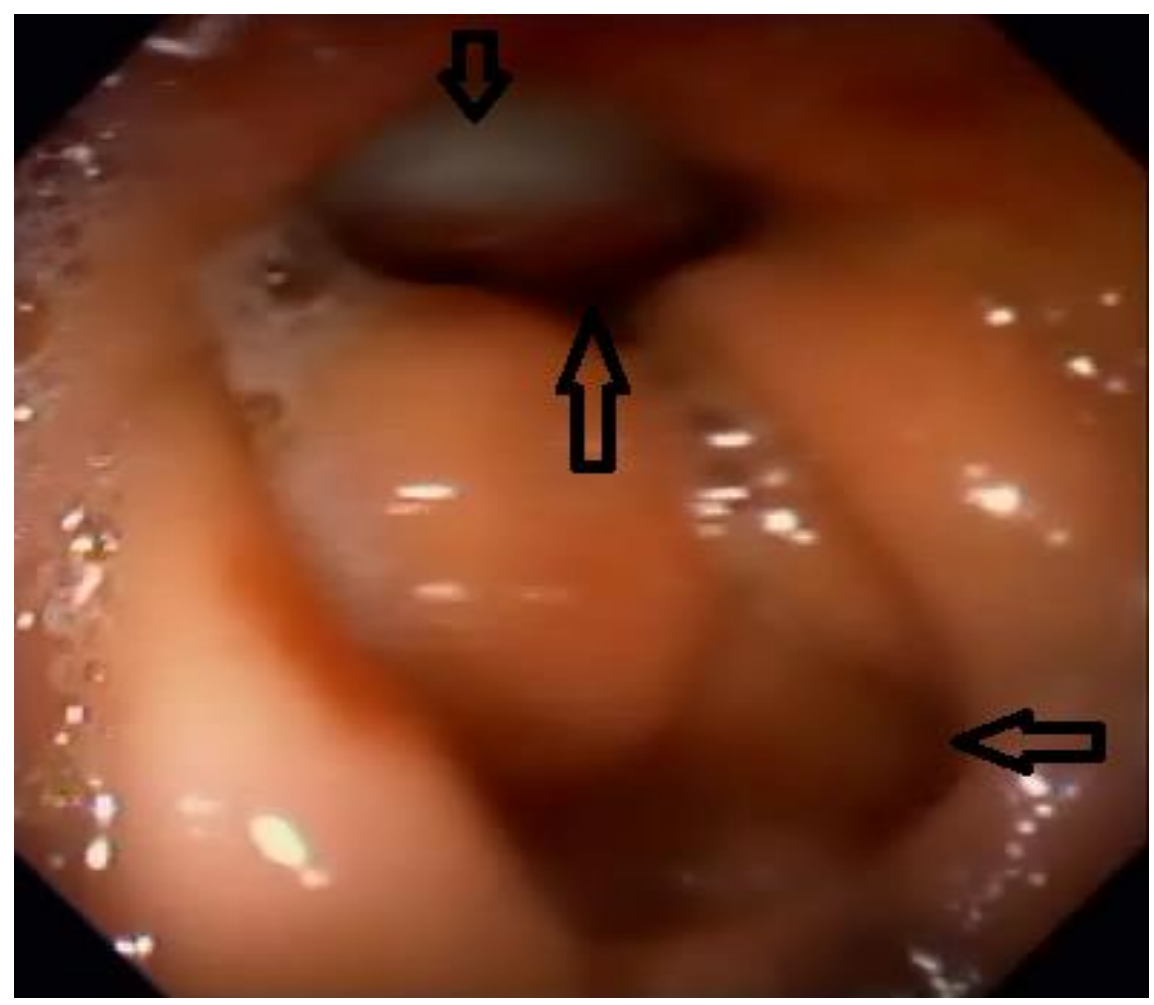

Fig. 1. Gastroscopy image revealed a $3 \mathrm{~cm}$ by $2 \mathrm{~cm}$ gastric diverticulum (up and down arrows) which contained an ulcer in its wall (down arrow) in close proximity of the opening of the unobstructed pylorus (right arrow). Also note the swelling of the surrounding mucosa.

\section{Case Presentation}

A 59-year-old gentleman presented with a history of epigastric pain and vomiting of copious amounts of blood. He also noted melena stools. Stabilization had included transfusion with 2 units of blood as the patient presented in cardiovascular decompensation and had a packed cell volume of $14 \%$.
The patient is a non-smoker who had regularly been ingesting non-steroidal anti-inflammatory drugs (NSAIDs) for the relief of chronic back pains that he frequently suffered from.

A subsequent gastroscopy revealed a $3 \mathrm{~cm}$ by 2 $\mathrm{cm}$ shallow diverticulum in close proximity of the opening of the pylorus-which was not obstructed in any way- the appearance was reminiscent of 
a 'double pylorus' (Fig. 1). The lining of the diverticulum of the featured an ulcer crater with its base lined with whitish inflammatory tissue admixed with blood clots. The base of this ulcer was filled with whitish inflammatory tissue and visible blood clots which were quiescent during the procedure (Forrest Class lllb). No other significant abnormality was noted.

The edge of the ulcer was biopsied. The results from the histopathology labs stated that the sample contained stomach ulcerations with severely active, chronic inflammatory gastritis. The samples were, however, free of Helicobacter pylori colonization and any suggestions of dysplasia or malignancy.

His clotting parameters were normal and he had remarkable response to the initial resuscitation measures, discontinuation of injurious drugs and proton pump inhibitor therapy. Hence, the decision was made to avoid surgical intervention. He was discharged in stable condition within a week of admission and has since avoided NSAID use altogether.

A follow up endoscopy, done 8 weeks on revealed that the ulcer had healed nicely and the patient has been symptom free 3 years afterwards.

\section{Discussion}

The acquired variety of GD is well documented to be caused by another gastrointestinal pathology. Based on their pathophysiologic origins, they are further divided into the pulsation type, which may follow conditions associated with increased intraluminal pressure (e.g. chronic coughing, difficult labour, pyloric obstruction) or from the eroding effect of gastric ulcers or carcinoma and the traction type, due to perigastric adhesions resulting from inflammatory lesions of the spleen, gallbladder, pancreas, liver, peritoneum. They could also follow gastric surgical procedures, e.g. Roux-enY gastric bypass ${ }^{6}$. The congenital variant of GD, on the other hand, is as a result of an underlying weakness in the gastric wall resulting from an anomaly at embryogenesis 7,8 .
Endoscopy is rapidly becoming the major tool for diagnosing many a $\mathrm{GD}^{2,6}$. Apart from enabling direct GD visualization, this diagnostic modality also allows one to detect other pathologies ${ }^{6}$. This point is important as reflected by the index case report where a huge ulcer crater in the pylorus was the site responsible for the bleeding. The use of endoscopic investigations has expanded to intraoperative localization of the diverticulum with placement of the scope or surgical clamp inside the deformity ${ }^{6}$. Note is made of the fact that no attempt was made to dislodge the clots, as per professional guidelines, seeing we had no facility to endoscopically deal with a consequent bleed ${ }^{9}$.

This major mode of GD identification is in contrast to earlier times when barium studies was the main method of diagnosis ${ }^{10}$. Abdominal imaging with computed tomography (CT) is also useful to delineate a GD's anatomic relations but confusion with adrenal masses, splenic abnormalities or pancreatic tail lesions has been reported ${ }^{11}$. Hence, it is advisable to give oral contrast to facilitate diagnosing GD during $\mathrm{CT}$ scanning ${ }^{6}$.

Clinically, GDs are mostly asymptomatic and as such, need no specific treatment. This is even truer as it relates to the congenital type as this is known to rarely cause symptoms. The acquired type would present with a background history suggestive of the inciting GIT pathology, such as peptic ulcer disease, malignancy, pancreatitis, or gastric outlet obstruction ${ }^{2}$. In the majority of asymptomatic cases conservative management can be the first approach-as was the case in this presented patient, where the decision was made not to do anything about the GD, because it had not caused him any symptoms previously. However, active medical therapy was instituted for the pyloric ulcer.

Surgical resection is reserved for when the GD is large, symptomatic or complicated by bleeding, perforation or malignancy and this is necessary in only about $10 \%$ of patients ${ }^{6,12}$. In between these poles, there are various medical therapies that have been shown to be effective 
with or without endoscopic ${ }^{12}$. The index case in this report responded well to conservative therapy. He was informed of the need to avoid further insults to the lining of the diverticulum which could spell catastrophic consequences like perforations. He thus decided to avoid NSAIDs all together and has been without similar symptoms in 3 years.

This report seeks to highlight the important role of endoscopy in the management of such presentations especially from our resource limited environment. Around here, the facilities are either non-existent or access to them is limited. The unique coexistence with another pathology that was responsible for the bleeding was easily identified. Moreover, the procedure has perhaps spared the patient an unnecessary surgical intervention.

\section{Conclusion}

The uncommon endoscopic observation of a GD is hereby presented. The even rarer occurrence of GD in the prepylorus is also documented. The coexistence of a drug-pyloric ulcer in the wall of the GD is noted. The selected management modality was conservative and the patient fared well.

\section{References}

1. Akerlund D. Gastric diverticulum. Acta Radiol 1923;2:476-85.

2. Anaise D, Brand DL, Smith NL, Soroff HS. Pitfalls in the diagnosis and treatment of a symptomatic gastric diverticulum. Gastrointestinal Endoscopy 1984;30:28-30. doi: 10.1016/s00165107(84)72291-7

3. Gockel I, Thomschke D, Lorenz D. Gastrointestinal: Gastric diverticula. J Gastroenterol Hepatol 2004;19:227. doi: 10.1186/1749-7922-7-1

4. Schiller AH, Roggendorf B, Delker-Wegener S, Richter K, Kuthe A. Laparoscopic resection of gastric diverticula: two case reports. Zentralbl Chir 2007;132(3):251-5. doi: 10.1055/s-2007-960753

5. Meeroff M, Goll'an JRM, Meeroff JC. Gastric diverticulum. Am J Gastroenterol 1967;47(3):189_ 203. PMID: 4960419

6. Tsitsias T, Finch JG. Gastric Diverticulum of the Prepyloric Region: A Rare Presentation of Gastric Diverticulum. Case Rep Gastroenterol 2012;6:150-4. doi: 10.1159/000338067
7. Mohan $\mathrm{P}$, Ananthavadivelu M, Venkataraman J. Gastric Diverticulum. CMAJ 2010;182(5):226. doi: 10.1503/cmaj.090832

8. Cheng $\mathrm{EH}$, Pavelock RR. Multiple gastrointestinal tract diverticula. Gastrointest Radiol 1990;15:282-4. doi: 10.1007/BF01888797

9. Hwang $\mathrm{JH}$, Fisher DA, Ben-Menachem $\mathrm{T}$, Chandrasekhara V, Chathadi K, Decker GA, et al. The role of endoscopy in the management of acute non-variceal upper Gl bleeding. Gastrointest Endosc 2012;75(6):1132-8. doi.org/10.1016/j.gie.2012.02.033

10. Casberg MA, Martin WP: Gastric diverticula. Am J Surg 1948;76:172-181. doi: 10.1016/00029610(48)90069-5

11. Chasse $E$, Buggenhout $A$, Zalcman $M$, Jeanmart J, Gelin M, El Nakadi I. Gastric diverticulum simulating a left adrenal tumor. Surgery 2003;133:447-448. doi: 10.3892/ol.2015.3559

12. Rashid F, Aber A, Iftikhar SY. A review on gastric diverticulum. World J Emerg Surg 2012;7:1. doi: 10.1186/1749-7922-7-1

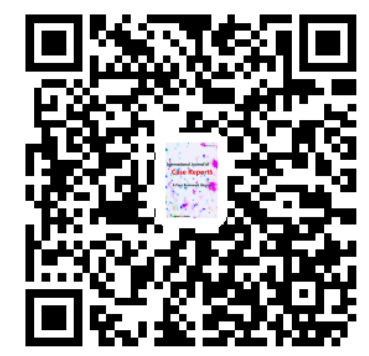

